\title{
Genome-wide survey and identification of AP2/ERF genes involved in shoot and leaf development in Liriodendron chinense
}

\author{
Yaxian Zong ${ }^{\dagger}$, Ziyuan $\mathrm{Hao}^{\dagger}$, Zhonghua Tu, Yufang Shen, Chengge Zhang, Shaoying Wen, Lichun Yang,
} Jikai Ma and Huogen Li

\begin{abstract}
Background: Liriodendron chinense is a distinctive ornamental tree species due to its unique leaves and tulip-like flowers. The discovery of genes involved in leaf development and morphogenesis is critical for uncovering the underlying genetic basis of these traits. Genes in the AP2/ERF family are recognized as plant-specific transcription factors that contribute to plant growth, hormone-induced development, ethylene response factors, and stress responses.

Results: In this study, we identified 104 putative AP2/ERF genes in the recently released L. chinense genome and transcriptome database. In addition, all 104 genes were grouped into four subfamilies, the AP2, ERF, RAV, and Soloist subfamilies. This classification was further supported by the results of gene structure and conserved motif analyses. Intriguingly, after application of a series test of cluster analysis, three AP2 genes, LCERF 94, LCERF 96, and LCERF 98, were identified as tissue-specific in buds based on the expression profiles of various tissues. These results were further validated via RT-qPCR assays and were highly consistent with the STC analysis. We further investigated the dynamic changes of immature leaves by dissecting fresh shoots into seven discontinuous periods, which were empirically identified as shoot apical meristem (SAM), leaf primordia and tender leaf developmental stages according to the anatomic structure. Subsequently, these three candidates were highly expressed in SAM and leaf primordia but rarely in tender leaves, indicating that they were mainly involved in early leaf development and morphogenesis. Moreover, these three genes displayed nuclear subcellular localizations through the transient transformation of tobacco epidermal cells.
\end{abstract}

Conclusions: Overall, we identified 104 AP2/ERF family members at the genome-wide level and discerned three candidate genes that might participate in the development and morphogenesis of the leaf primordium in $L$. chinense.

Keywords: Liriodendron chinense, AP2/ERF family, Transcription factors, Shoot-specific, Leaf development

\footnotetext{
* Correspondence: hgli@njfu.edu.cn

†Yaxian Zong and Ziyuan Hao contributed equally to this work

Co-Innovation Center for Sustainable Forestry in Southern China, Key

Laboratory of Forest Genetics and Biotechnology of Ministry of Education,

Nanjing Forestry University, Nanjing 210037, China
}

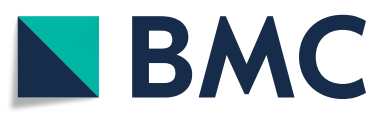

(c) The Author(s). 2021 Open Access This article is licensed under a Creative Commons Attribution 4.0 International License, which permits use, sharing, adaptation, distribution and reproduction in any medium or format, as long as you give appropriate credit to the original author(s) and the source, provide a link to the Creative Commons licence, and indicate if changes were made. The images or other third party material in this article are included in the article's Creative Commons licence, unless indicated otherwise in a credit line to the material. If material is not included in the article's Creative Commons licence and your intended use is not permitted by statutory regulation or exceeds the permitted use, you will need to obtain permission directly from the copyright holder. To view a copy of this licence, visit http://creativecommons.org/licenses/by/4.0/. The Creative Commons Public Domain Dedication waiver (http://creativecommons.org/publicdomain/zero/1.0/) applies to the data made available in this article, unless otherwise stated in a credit line to the data. 


\section{Background}

Plant morphogenesis is mainly related to the shoot as well as the activity of the shoot apical meristem (SAM) $[1,2]$, which further gives rise to stems, tender leaves, or other tissues and organs. Plant endogenous hormones are recognized to play a crucial role in regulating leaf development and morphogenesis [3, 4]. Moreover, functional dissections of plant transcription factors (TFs), such as NAC [5], KNOX [6], and AP2/ERF [7, 8], which have been largely described as being involved in shoot and leaf development, have been revealed from studies on Arabidopsis.

APETALA2/ethylene responsive element-binding proteins (AP2/EREBPs) are a well-known transcription family that has been reported to be involved in ethylene response, biotic or abiotic stress resistance, cell differentiation, cell expansion, and stress signaling pathways in plants [9-11]. As one of the largest TF families of plants, the AP2/ERF family is composed of four subfamilies with over 100 members in various taxa [12-14]. A feature of the AP2/ERF-type DNA binding domain, which consists of $60 \sim 70$ residues, is universally contained in the proteins of this family [15]. Moreover, on the basis of the types and quantities of conserved domains, the AP2/ERF family can be divided into the AP2, RAV, ERF, and Soloist subfamilies. Proteins of the AP2 subfamily contain two repetitive AP2 domains, while the ERF subfamily proteins have only a single AP2 domain. In the RAV subfamily, tandem AP2 and B3 domains were found in the primary protein sequences [16-18]. Finally, the Soloist subfamily has been historically regarded as an ERF member and is currently regrouped into a novel subfamily due to its single AP2 domain and strong sequence divergence $[16,19]$. In addition, based on the sequence similarity of the AP2/ERF motifs, the ERF family is further divided into the ERF and DREB subfamilies $[18,20]$.

Although four clades have been maintained in the AP2/ERF family, their functions largely depend on the interaction of their motifs with specific regulatory elements [16]. In general, the ERF and DREB subfamilies primarily function in resistance to biotic or abiotic stresses [21], and the "WLG" motif is considered a typical feature of these subfamilies [18]. Additionally, the DREB subfamily is mainly involved in improving abiotic stress tolerance in plants, including salinity stress, drought stress, water deficit, low temperature, and other abiotic stresses, via the interaction of a core motif of A/GCCGAC with downstream dehydration responsive elements (DREs) [22]. The ERF subfamily participates in defense by integrating the cisacting element AGCCGCC with its GCC box [23, 24]. However, this is not always the case, and this resistance can be interrupted by the VIII groups of these families [25]. DRN and DRNL genes in Arabidopsis hierarchically interact in auxin signaling and patterning of the apical embryo. In addition, $L E A F Y$ PETIOLE (LEP) acts as a positive regulator of gibberellic acid-induced germination and is involved in the formation of petioles [26]. The AP2 subfamily usually regulates the development of shoots as well as the stem cell niche during embryonic pattern formation $[27,28]$. In summary, these inferences from previous studies provide direction for comprehensively understanding AP2/ERFs and the discovery of novel genes involved in leaf and shoot development.

Liriodendron chinense (Hemsl.) Sarg. is a relict tree species that is native to southern China. It is famous for its odd leaf shape, which looks like a "riding jacket", and is widely used as an ornamental in landscapes and gardens. However, the underlying genetic mechanisms of leaf development and morphogenesis remain poorly understood. As a valuable ornamental plant, it is meaningful to understand the development and morphogenesis of $L$. chinense leaves. Along with the recent release of the $L$. chinense genome and transcriptome information [29-31], we have new insights for investigating gene families and exploring candidate genes involved in SAM and leaf development.

In this study, we identified 104 LcAP2/ERF genes by combining a genome-wide survey and transcription data of various tissues. Moreover, we described the conserved motif, gene structure, and phylogenetic analyses and divided the LcAP2/ERF genes into four subfamilies of approximately 14 groups. Through expression profile analysis and series test of cluster (STC) analysis, we discovered the genes expressed specifically in shoots and further examined the expression patterns of these candidates dynamically in the different developmental stages. This work will lay a foundation for the comprehensive understanding of the LcAP2/ERF family and will also be helpful for determining candidate genes involved in leaf development in L. chinense.

\section{Results \\ Identification of AP2/ERF TFs in L. chinense}

Based on the HMM profiles (PF00847) and homology searches, a total of 104 putative AP2/ERF genes designated LcERF1 to LcERF104 were identified in L. chinense. All these candidates contained one or more AP2/ERF domains according to conservative domain analysis. Then, we described the characteristics of their proteins, including the coding sequence (CDS) length, protein length, molecular weight $(\mathrm{MW})$, isoelectric point (PI), and predicted subcellular localization (see Additional file 1: Table S1). Accordingly, the protein lengths of these 104 AP2/ERFs ranged from 100 aa 
Table 1 List of the 104 AP2/ERF genes identified in Liriodendron Chinense

\begin{tabular}{|c|c|c|c|c|c|}
\hline Gene name & Gene ID & Location & Protein length (aa) & Introns & Family group \\
\hline LCERF1 & Unigene40981_All & Scaffold211 & 261 & 0 & I \\
\hline LCERF2 & Lchi03057 & Scaffold506 & 328 & 1 & I \\
\hline LCERF3 & Lchi07965 & Scaffold708 & 325 & 1 & । \\
\hline LCERF4 & Lchi07966 & Scaffold708 & 561 & 2 & I \\
\hline LCERF5 & Lchi22931 & Scaffold1519 & 432 & 1 & 1 \\
\hline LCERF6 & Lchi09796 & Scaffold2048 & 316 & 1 & I \\
\hline LCERF7 & Lchi16995 & Scaffold3097 & 316 & 0 & I \\
\hline LCERF8 & Lchi23250 & Scaffold142 & 152 & 0 & $\|$ \\
\hline LCERF9 & Lchi16170 & Scaffold408 & 193 & 1 & $\|$ \\
\hline LCERF10 & Unigene12650_All & Scaffold416 & 188 & 0 & $\|$ \\
\hline LCERF11 & Lchi16911 & Scaffold480 & 199 & 1 & $\|$ \\
\hline LCERF12 & Unigene40401_All & Scaffold525 & 185 & 0 & $\|$ \\
\hline LCERF13 & Unigene20830_All & Scaffold836 & 186 & 0 & $\|$ \\
\hline LCERF14 & Lchi11957 & Scaffold345 & 224 & 1 & III \\
\hline LCERF15 & Lchi04946 & Scaffold530 & 235 & 1 & III \\
\hline LCERF16 & Lchi04947 & Scaffold530 & 295 & 1 & III \\
\hline LCERF17 & CL2522.Contig2_All & Scaffold530 & 223 & 0 & III \\
\hline LCERF18 & CL10877.Contig3_All & Scaffold530 & 223 & 0 & III \\
\hline LCERF19 & Unigene6126_All & Scaffold530 & 211 & 0 & III \\
\hline LCERF20 & Lchi33109 & Scaffold1203 & 229 & 1 & III \\
\hline LCERF21 & Lchi33111 & Scaffold1203 & 227 & 1 & III \\
\hline LCERF22 & Lchi34895 & Scaffold1374 & 513 & 4 & III \\
\hline LCERF23 & Lchi29925 & Scaffold1675 & 425 & 3 & III \\
\hline LCERF24 & Lchi08587 & Scaffold39 & 420 & 1 & III \\
\hline LCERF25 & CL5589.Contig2_All & Scaffold345 & 203 & 0 & III \\
\hline LCERF26 & Unigene11386_All & Scaffold432 & 211 & 0 & III \\
\hline LcERF27 & CL5589.Contig1_All & Scaffold530 & 246 & 0 & III \\
\hline LCERF28 & Lchi00950 & Scaffold723 & 217 & 0 & III \\
\hline LCERF29 & Lchi01616 & Scaffold1191 & 100 & 1 & III \\
\hline LCERF30 & Unigene5530_All & Scaffold1191 & 252 & 0 & III \\
\hline LCERF31 & Lchi32377 & Scaffold1289 & 210 & 0 & III \\
\hline LCERF32 & Lchi26370 & Scaffold1364 & 193 & 1 & III \\
\hline LCERF33 & Lchi08922 & Scaffold3419 & 244 & 0 & III \\
\hline LCERF34 & Lchi28169 & Scaffold654 & 418 & 1 & IV \\
\hline LCERF35 & Lchi23878 & Scaffold1043 & 141 & 0 & IV \\
\hline LcERF36 & Lchi22387 & Scaffold1263 & 229 & 1 & IV \\
\hline LcERF37 & Lchi13652 & Scaffold1315 & 429 & 1 & IV \\
\hline LCERF38 & Lchi30363 & Scaffold2365 & 475 & 3 & IV \\
\hline LCERF39 & Lchi30365 & Scaffold2365 & 354 & 1 & IV \\
\hline LCERF40 & Lchi31374 & Scaffold3032 & 404 & 1 & IV \\
\hline LCERF41 & Lchi34724 & Scaffold3708 & 355 & 1 & IV \\
\hline LcERF42 & Lchi10868 & Scaffold159 & 758 & 2 & V \\
\hline LCERF43 & Lchi11945 & Scaffold345 & 421 & 7 & V \\
\hline LCERF44 & Lchi25937 & Scaffold1371 & 183 & 1 & V \\
\hline
\end{tabular}


Table 1 List of the 104 AP2/ERF genes identified in Liriodendron Chinense (Continued)

\begin{tabular}{|c|c|c|c|c|c|}
\hline Gene name & Gene ID & Location & Protein length (aa) & Introns & Family group \\
\hline LCERF45 & Lchi16637 & Scaffold2432 & 226 & 1 & V \\
\hline LCERF46 & Lchi34468 & Scaffold2926 & 206 & 1 & V \\
\hline LCERF47 & Lchi05084 & Scaffold3476 & 136 & 1 & V \\
\hline LCERF48 & Lchi07311 & Scaffold 172 & 268 & 0 & $\mathrm{Vl}$ \\
\hline LCERF49 & Lchi22103 & Scaffold920 & 369 & 2 & $\mathrm{Vl}$ \\
\hline LCERF50 & Lchi17039 & Scaffold3097 & 359 & 1 & $\mathrm{Vl}$ \\
\hline LCERF51 & Lchi02638 & Scaffold416 & 310 & 1 & VII \\
\hline LCERF52 & Lchi02639 & Scaffold416 & 362 & 1 & VII \\
\hline LcERF53 & Lchi11452 & Scaffold525 & 383 & 1 & VII \\
\hline LCERF54 & Lchi04620 & Scaffold775 & 290 & 1 & VII \\
\hline LcERF55 & Lchi04621 & Scaffold775 & 289 & 1 & VII \\
\hline LcERF56 & Lchi04623 & Scaffold775 & 236 & 1 & VII \\
\hline LcERF57 & Lchi07083 & Scaffold135 & 273 & 1 & VIII \\
\hline LcERF58 & Lchi07084 & Scaffold135 & 203 & 0 & VIII \\
\hline LcERF59 & Lchi13371 & Scaffold1075 & 207 & 1 & VIII \\
\hline LcERF60 & Lchi11824 & Scaffold1130 & 375 & 3 & VIII \\
\hline LcERF61 & Lchi13392 & Scaffold1763 & 207 & 1 & VIII \\
\hline LcERF62 & Unigene7795_All & Scaffold1763 & 205 & 0 & VIII \\
\hline LcERF63 & Lchi31572 & Scaffold1784 & 180 & 1 & VIII \\
\hline LcERF64 & Lchi08484 & Scaffold39 & 204 & 1 & IX \\
\hline LcERF65 & Lchi09908 & Scaffold79 & 316 & 1 & IX \\
\hline LcERF66 & Unigene24905_All & Scaffold79 & 170 & 0 & IX \\
\hline LcERF67 & Lchi01406 & Scaffold432 & 211 & 1 & IX \\
\hline LcERF68 & Unigene35921_All & Scaffold432 & 301 & 0 & IX \\
\hline LcERF69 & Lchi08172 & Scaffold580 & 324 & 0 & IX \\
\hline LCERF70 & Lchi07909 & Scaffold708 & 174 & 1 & IX \\
\hline LCERF71 & Lchi31530 & Scaffold803 & 105 & 2 & IX \\
\hline LcERF72 & CL9762.Contig1_All & Scaffold1024 & 307 & 0 & IX \\
\hline LcERF73 & Lchi05992 & Scaffold1024 & 250 & 1 & IX \\
\hline LCERF74 & Lchi05993 & Scaffold1024 & 350 & 2 & IX \\
\hline LcERF75 & Lchi26525 & Scaffold1934 & 272 & 1 & IX \\
\hline LcERF76 & Lchi26532 & Scaffold1934 & 361 & 3 & IX \\
\hline LcERF77 & Lchi28702 & Scaffold54 & 309 & 2 & $x$ \\
\hline LCERF78 & Unigene10666_All & Scaffold 100 & 235 & 1 & $x$ \\
\hline LCERF79 & Lchi02215 & Scaffold682 & 229 & 1 & $x$ \\
\hline LCERF80 & Lchi02216 & Scaffold 682 & 196 & 1 & $x$ \\
\hline LCERF81 & Lchi18461 & Scaffold943 & 403 & 1 & $x$ \\
\hline LCERF82 & Lchi11856 & Scaffold1130 & 128 & 1 & $x$ \\
\hline LcERF83 & Lchi01932 & Scaffold1191 & 292 & 1 & $x$ \\
\hline LcERF84 & Lchi20453 & Scaffold1167 & 328 & 2 & VI-L \\
\hline LcERF85 & Lchi14855 & Scaffold41 & 394 & 6 & AP2 \\
\hline LCERF86 & Lchi23120 & Scaffold192 & 405 & 7 & AP2 \\
\hline LCERF87 & Lchi16948 & Scaffold480 & 550 & 9 & AP2 \\
\hline LCERF88 & Lchi08043 & Scaffold502 & 680 & 6 & AP2 \\
\hline
\end{tabular}


Table 1 List of the 104 AP2/ERF genes identified in Liriodendron Chinense (Continued)

\begin{tabular}{|c|c|c|c|c|c|}
\hline Gene name & Gene ID & Location & Protein length (aa) & Introns & Family group \\
\hline LCERF89 & Lchi11241 & Scaffold503 & 375 & 8 & AP2 \\
\hline LCERF90 & Lchi28881 & Scaffold509 & 474 & 7 & AP2 \\
\hline LCERF91 & Lchi06162 & Scaffold527 & 524 & 7 & AP2 \\
\hline LCERF92 & Lchi03252 & Scaffold764 & 535 & 6 & AP2 \\
\hline LCERF93 & CL7987.Contig2_All & Scaffold805 & 572 & 12 & AP2 \\
\hline LCERF94 & Unigene5404_All & Scaffold2118 & 490 & 6 & AP2 \\
\hline LCERF95 & Lchi33401 & Scaffold2225 & 563 & 6 & AP2 \\
\hline LCERF96 & Lchi13837 & Scaffold2467 & 662 & 7 & AP2 \\
\hline LcERF97 & CL6967.Contig2_All & Scaffold2956 & 327 & 6 & AP2 \\
\hline LCERF98 & Unigene39546_All & Scaffold3476 & 468 & 7 & AP2 \\
\hline LcERF99 & Lchi08779 & Scaffold67 & 376 & 1 & RAV \\
\hline LCERF100 & Lchi02516 & Scaffold100 & 607 & 4 & RAV \\
\hline LCERF101 & Lchi02519 & Scaffold100 & 428 & 2 & RAV \\
\hline LCERF102 & Lchi15640 & Scaffold1242 & 354 & 2 & RAV \\
\hline LCERF103 & Lchi23744 & Scaffold1330 & 361 & 1 & RAV \\
\hline LCERF104 & Lchi32356 & Scaffold3563 & 235 & 5 & Soloist \\
\hline
\end{tabular}

(LcERF29) to 758 aa (LcERF42), with an average of approximately 317 aa (Table 1). Moreover, the molecular weights of the proteins varied from $11.48 \mathrm{kDa}$ (LcERF29) to $84.42 \mathrm{KDa}$ (LcERF42). In addition, the isoelectric point values of these proteins ranged from 4.72 (LcERF27) to 10.22 (LcERF67). The subcellular localization predicted that 83 LcERF proteins were located in the nuclear region, 13 LcERF proteins were located in the chloroplast region, and the remaining genes were distributed in the cytoplasm, mitochondria, plasma membrane, and other areas (see Additional file 1: Table S1).

\section{Phylogenetic analysis and classification of LcERF genes}

Based on conservative domain analysis and multiple alignments of LcERF protein sequences, the 104 LcERF proteins were categorized into four subfamilies, including the ERF, AP2, RAV, and Soloist subfamilies. All 84 ERF proteins contained a single AP2 domain, and based on the characteristics of the amino acid sequences and domains that they encode, these genes were further divided into two subfamilies, which were named the DREB and ERF subfamilies and covered 41 and 43 members, respectively. However, among the remaining genes, 14 genes were identified as members of the AP2 family owing to their tandemly repeated double AP2/ERF domain. In addition, 5 genes that not only possessed a single AP2/ERF domain but also displayed a B3 domain were classified in the RAV subfamily. The last one, LcERF104, is homologous to the Arabidopsis Soloist gene (At4g13040) and was classified in the Soloist subfamily. According to the description in Nakano's study [19], the DREB subfamily comprises four parts, named I, II, III, and IV, which contain 7, 6, 20, and 8 members, respectively. The ERF subfamily genes can be divided into seven groups based on phylogenetic analysis and belong to the V, VI, VII, VIII, IX, X, and VI-L parts with $6,3,6,7,13,7$, and 1 members, respectively. The sequence alignment of LcERF proteins showed that the WLG element was highly conserved in the ERF, DREB and RAV subfamilies but less conserved in the AP2 subfamily. However, RAYD, AA, and other elements were conserved in the AP2 subfamily (Fig. 1).

The evolutionary relationships of all candidate genes were further illustrated by phylogenetic analysis. According to the unrooted tree profile, AP2, RAV, and Soloist were clustered in a separate branch within the subfamily. However, ERF subfamily were divided into 2 large branches, the ERF branch and the DREB branch, and the ERF and DREB branches were divided into 7 and 4 groups, respectively (Fig. 2). Moreover, these findings coincided with the grouping of the ERF subfamily described above based on the conserved motifs (Fig. 1). In addition, this result showed the same clustering pattern as that obtained by the classification method based on alignment with Arabidopsis (Table 1). As a result, we propose that these 104 putative genes are indeed AP2/ EFR family genes in L. chinense.

\section{Gene structure and conserved motif analysis}

To further understand the structural composition of LcERF genes, we analyzed the genomic DNA sequence using the online Gene Structure Display Server, with the locations of exons and introns provided by the $L$. 


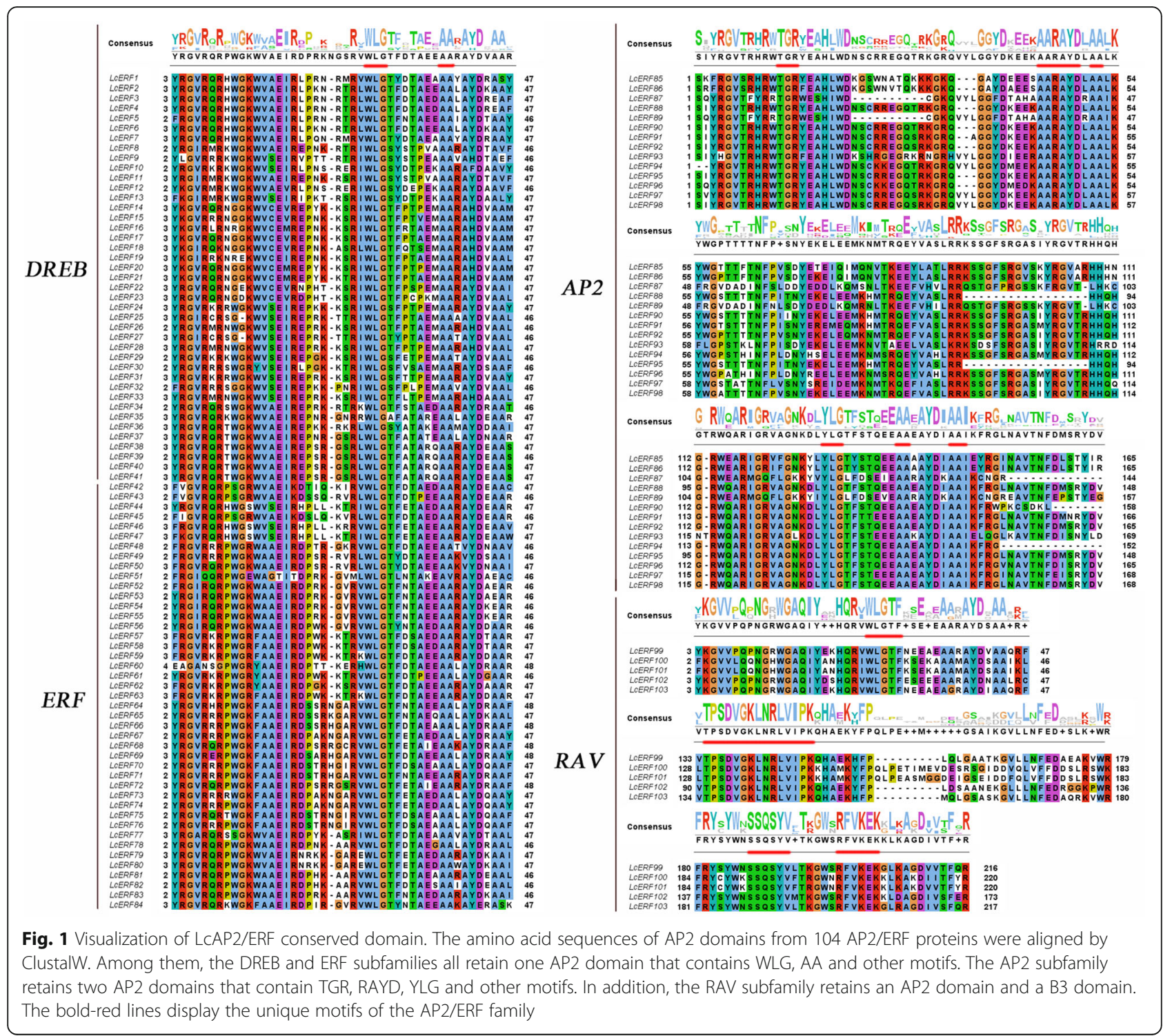

chinense genomic resource. According to the structural characteristics of LcERF genes, the number of introns varied among the distinct subfamilies (Fig. 3A). Except for a few members carrying more than one intron, most of the DREB and ERF subfamily genes had only one intron or even no introns in their genomic DNA. In the AP2 subfamily, all the genes possessed numerous introns, with intron numbers ranging from 6 to 12. Furthermore, LCERF93 was considered to have the most introns with 12, even though most AP2 genes contained 6 or 7 introns. Moreover, four of the five RAV members possessed one or two introns, and the single Soloist member contained five introns (Fig. 3B). In addition, the position of introns also presented interesting differences among different subfamilies. Concerning the sequences with an intron, the position of their intron was mostly near the $\mathrm{N}$-terminus or $\mathrm{C}$-terminus and rarely in the middle of the sequence because these sequences usually consisted of a long and a very short exon. In general, the members with close evolutionary relationships and from the same subfamily had similar exon and intron structures in terms of intron number and position and exon length.

Conserved motifs of 104 LcERF genes were identified using the MEME (Multiple Em for Motif Elicitation) tool. A total of 15 conserved motifs were displayed in the 104 LcERF proteins (Fig. 3C). The amino acid length of the 15 motifs ranged from 15 to 50 . Even though all of them had the AP2 domain, the protein sequences of the domain differed between the ERF and AP2 subfamilies. As AP2 DNA-binding motifs, motif 1 and motif 2 joined together and appeared in both the DREB and ERF subfamilies, except for special cases of motif 2, which also existed independently in the RAV and Soloist 


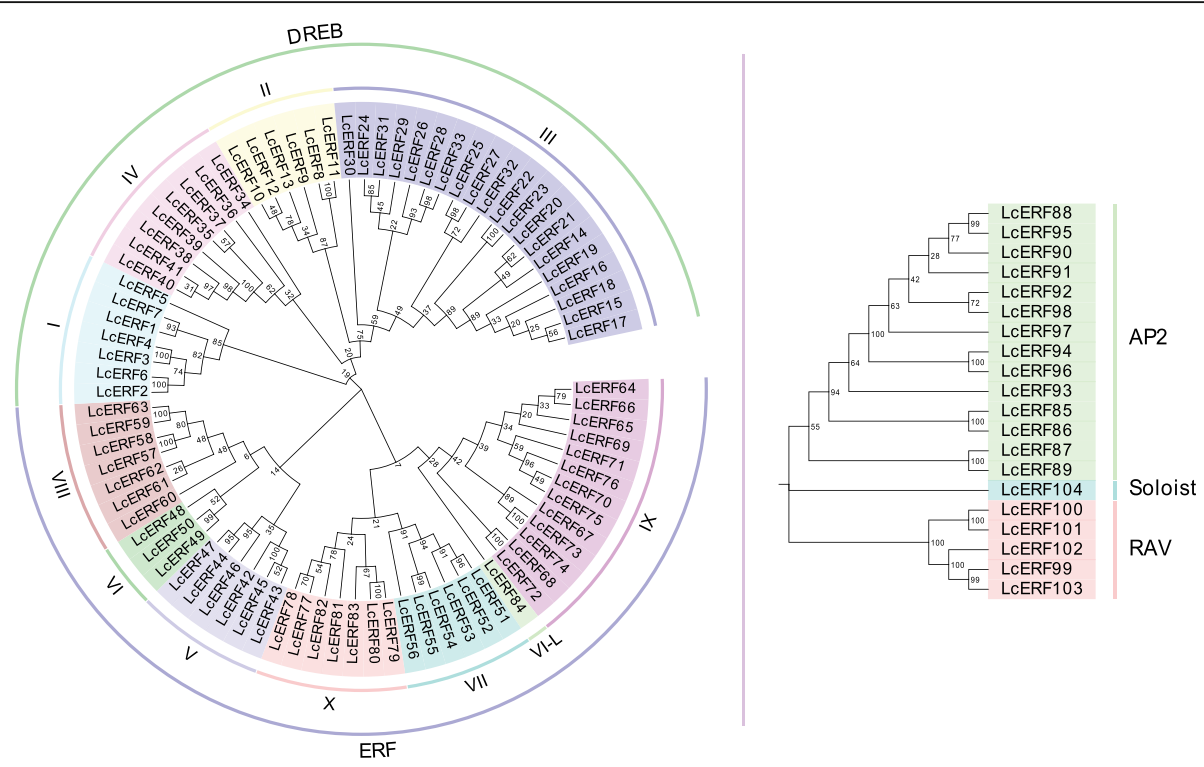

Fig. 2 Phylogenetic tree using the NJ method displaying the relationships among the AP2/ERF family of L. chinense. The phylogenetic tree was constructed using AP2/ERF domain sequences by the NJ method with 1000 bootstrap replicates in MEGA 7.0. The evolutionary distances were computed using the Poisson model. All 104 LcERF proteins were categorized into four subfamilies, including the ERF, AP2, RAV, and Soloist subfamilies. The ERF subfamily members were further divided into DREB subfamily (Groups II II, III, IV) and ERF subfamily (Groups V, VI, VII, VIII, IX, X, VI-L)

subfamilies. In the AP2 subfamily, the AP2 domain consisted of motif 3 and motif 6 , and some of them also contained motif 4 and motif 12 . In addition, although most of the ERF subfamily members shared the two conserved motifs of motif 1 and motif 2 , the other motifs varied in the different proteins. In the DREB subfamily, the proteins contained relatively more conserved motifs than in the ERF subfamily, especially in group III and group IV. Motifs 5, 7, 8, and 15 were detected in some group III proteins, and motifs $9,10,11$, and 12 were found in most group IV members. Moreover, motifs 13 and 14 only existed in the B3 domain and were also considered specific to the RAV subfamily.

\section{Expression profiles of LcERF genes in different tissues}

We investigated the expression profiles of LcERF genes in various tissues by Illumina RNA-Seq data [31] and constructed a heatmap, revealing that 86 LcERF genes were detected in the various tissues, including 34 genes in the DREB subfamily, 35 genes in the ERF subfamily, 12 genes in the AP2 subfamily, 4 genes in the RAV subfamily, and one Soloist gene. To explore the differential expression of these genes in different tissues, the FPKM values were standardized by row with TBtools software. Then, the standardized results were clustered by row and column (Fig. 4A). The results showed that several genes were expressed in all tissues and clustered in a large group. In addition, the column cluster divided the other genes based on their different expression patterns, including pistil-specific, stamen-specific, leaf-specific, shoot-specific, and other patterns.

\section{Expression patterns of LcERF genes and discovery of shoot-specific genes}

To reveal genes involved in shoot and leaf development, we intentionally focused on genes that were expressed specifically in the shoot tissue. All the $L C E R F$ family genes were divided into ten clusters in accordance with the K-means method in STEM software. Accordingly, cluster IV and cluster V showed tissue-specific expression in leaves and shoots, respectively. Cluster V contained eight genes, while cluster IV contained only one (Fig. 5A). We further tested the results of STC analysis of all the LcAP2/ ERF groups with an adjusted $p$-value $(p \leq 0.05)$, and only six of eight genes were significantly clustered in the $\mathrm{V}$ cluster, while the single member of cluster IV failed to pass the significance test (Fig. 5B). Among these six genes, three are ERF VIII members (LCERF57, LcERF58, and LcERF63), and another three belong to the AP2 subfamily (LcERF94, LcERF96, and LCERF98).

The expression of the six candidate genes showing shoot-specific patterns was further verified using RTqPCR. We determined the expression of these six genes in seven tissues: leaf, shoot, sepal, petal, stamen, pistil, and stem. Consistent with the STC results, the LcERF94, LCERF96, and LcERF98 genes were primarily expressed in shoots. While the expression of LcERF63 in different 

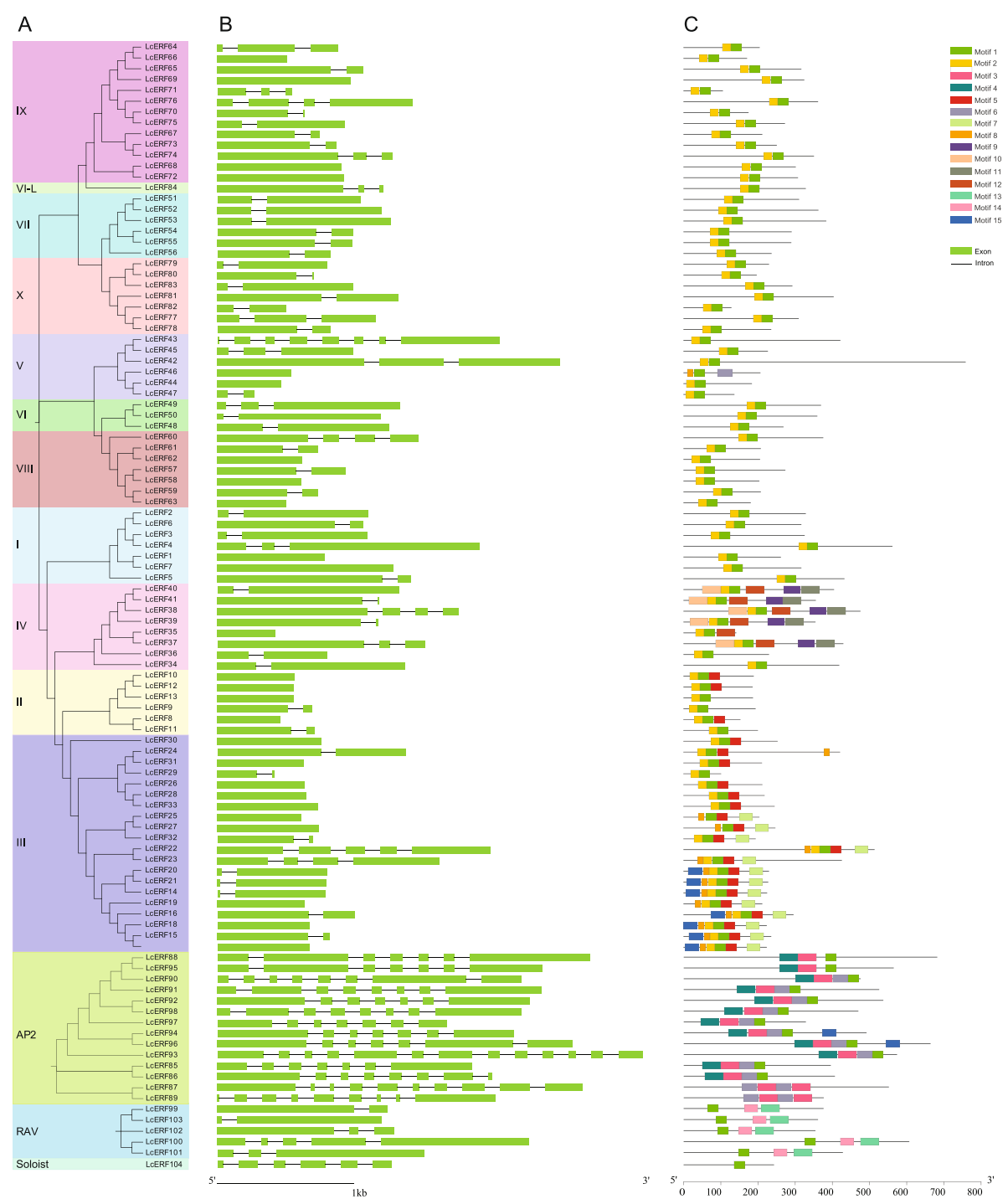

Fig. 3 Gene structure and conserved motif analysis of 104 LcERF genes. (A) Phylogenetic tree of 104 LcERF proteins. (B) Exon-intron structures of LCERF genes. Green boxes represent exons, and black lines represent introns. (C) Conserved motifs of LCERF proteins. The motifs, numbers 1-15, are displayed in different colored boxes

tissues was not specific, it was detected in both shoots and flowers, and LcERF58 was detected in all tissues (Fig. 4B). Unexpectedly, we failed to detect the LcERF57 genes even with repetitive optimization of the primer design and amplification conditions; hence, we did not perform the following assay of this gene. Considering the potential functions annotated in the NCBI GenBank and Gene Ontology (GO) databases, dual roles may exist for these three genes in Group VIII, as we inferred. Moreover, this conjecture has been clarified in previous studies, which have confirmed that ERF VIII subgroup genes play an important role in vitro shoot regeneration and development [32].

We then annotated the functions of these six genes by submitting sequences to TAIR (The Arabidopsis
Information Resource). Through alignment and annotation, all three genes of the AP2 subfamily were annotated as the AINTEGUMENTA (ANT) or AINTEGUMENTA-like (AIL) gene, which is also considered to be involved in maintenance of the shoot apical meristem (GO:0010492), the auxin-mediated signaling pathway involved in phyllotactic patterning (GO:0060774), plant organ morphogenesis (GO: 1905392), cell division (GO:0051301), and cell growth (GO:0016049). However, LcERF57, LcERF58, and LcERF63 from the VIII group were annotated with different functions, such as negative regulation of the ethylene-activated signaling pathway (GO: 0010105, GO:0009873) and glucosinolate metabolic process (GO:0019760). 


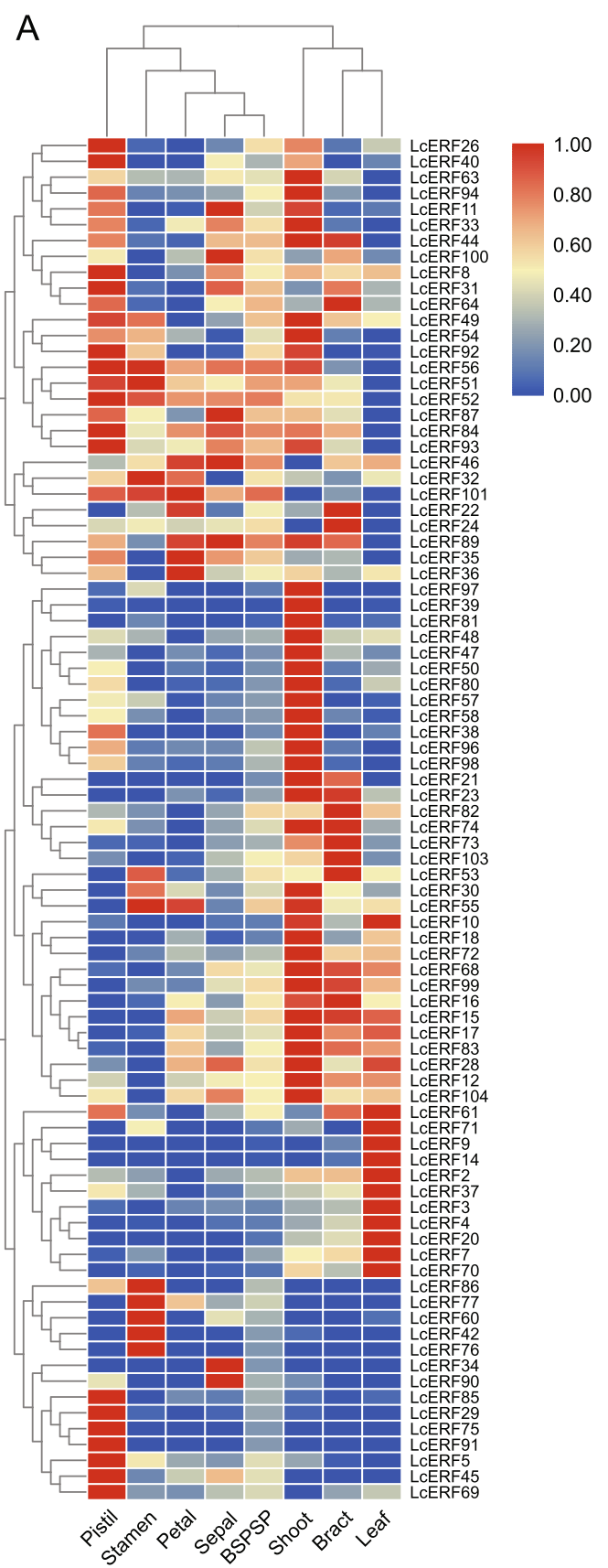

B
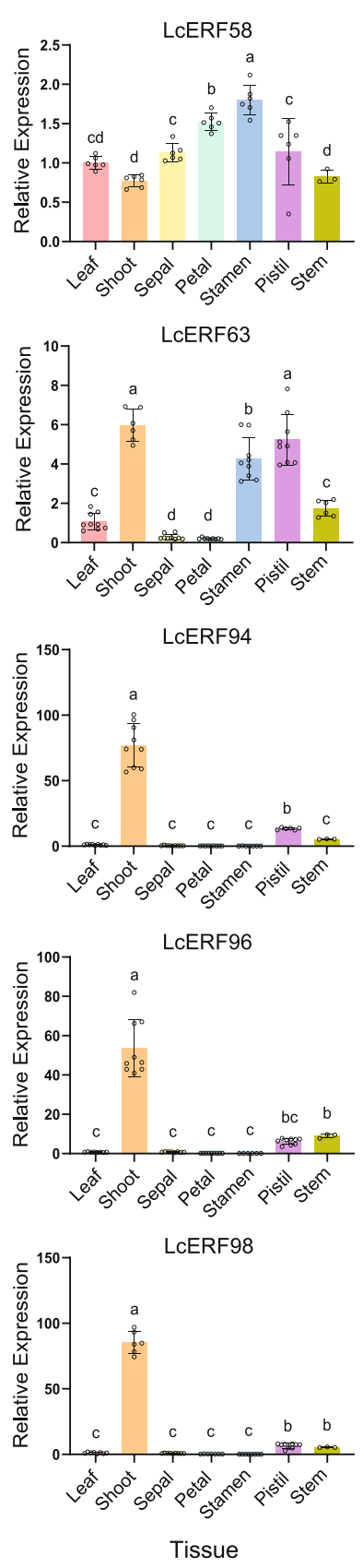

Fig. 4 Expression patterns of LCERFs in different tissues. (A) Hierarchical clustering of the expression profiles of 86 LcERFs in various tissues. The FPKM value was normalized by the Z-score algorithm. (B) Relative expression of five potential genes in various tissues. The round dot is the original value, and the error bar shows Std. deviation. For statistical analyses, ANOVA and Duncan tests were used $(p<0.05)$

Potential roles of shoot-specific candidates involved in shoot and leaf development

It is generally known that young leaves rise from shoots, and we further dissected the anatomical structure of the shoot layer by layer. In vitro shoots were divided into multiple layers of tender leaves, and discontinuous developmental stages of leaves, i.e., P0 (SAM), $\mathrm{P}_{1}, \mathrm{P}_{2}, \mathrm{P}_{3}, \mathrm{P}_{4}, \mathrm{P}_{5}$, and $\mathrm{P}_{6}$, are displayed in
Fig. 6A. Referring to the nomenclature, P1 and P2 periods were identified as leaf primordia, while P3 P6 were tender leaves when checking the anatomical structure. Leaf morphology and leaf lobes formed during the P1 and P2 stages, and the leaf size gradually increased from P3 to P6 (tender leaves), while SAM was the primary contributor to primordium differentiation in $L$. chinense leaves. 

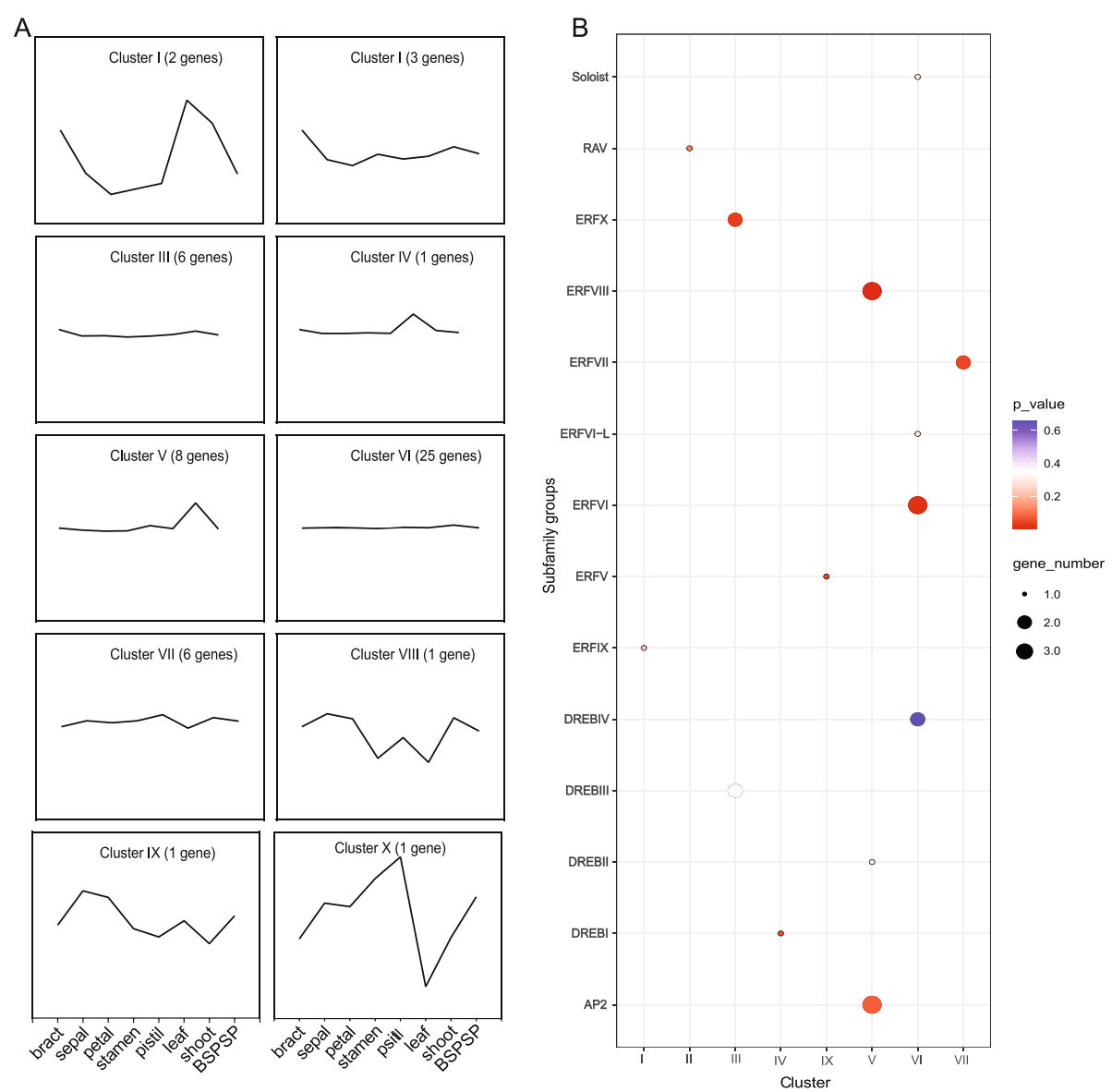

Fig. 5 Series test of cluster analysis of LCERF genes. (A) Ten clusters of all 86 LCERFs using the K-means method. The black line represents the trend of gene expression in different tissues. (B) Cluster detection of different subfamilies of LcERFs. The size of the circle represents the number of genes, and the color represents the $p$-value

Attracted by the shoot-specific patterns of the candidate genes, we further explored the potential functions of the candidates by detecting the expression from $\mathrm{P} 0$ to P6. RT-qPCR was performed to detect the expression of target genes from P1 to P6 as well as SAM (Fig. 6B). The results revealed that the expression of these genes decreased gradually from P0 to P6. Specifically, a significant difference was detected in the expression of the leaf primordia (P1-P2) and tender leaves (P3-P6) when examining the LcERF94, LcERF96, and LcERF98 genes; however, adverse consequences were found in comparisons of the SAM (P0) and leaf primordia (P1-P2). Taken together, this evidence supports $L c E R F 94 / 96 / 98$ genes as potential candidates involved in the early-stage development of leaf morphology in L. chinense.

\section{Subcellular localization of LcERF genes}

To investigate the potential function of these AP2 genes in transcriptional regulation, we detected the subcellular localization of LcERF94/96/98 using young tobacco leaves. Confocal microscopy was used to observe and photograph the transient transformed lower epidermal cells of tobacco leaves, and visible, GFP fluorescence, chlorophyll fluorescence, and merged field images were obtained (Fig. 7). The 35S::GFP, as a control sample, showed GFP fluorescence in the whole cell. The GFP fluorescence of pBI121-35S::GFP -LcERF94/96/98 was observed only in the nucleus, which is consistent with the characteristics of TFs, and these histological observations indicated that LcERF94/96/98 might function as TFs in the nucleus.

\section{Discussion}

The AP2/ERF transcription factors are ubiquitous in plant species and act as vital regulators governing various biological processes. They have been studied in many species, and the total number of AP2/ERF family genes varies among different plants, for example, 147 in A. thaliana [18, 19], 163 in rice $[19,21], 132$ in grape [33], and 200 in Populus trichocarpa [34] et al. [14, 3538]. Based on the features of the conserved domain, the AP2/ERF TFs are classified into four groups as ERF, 


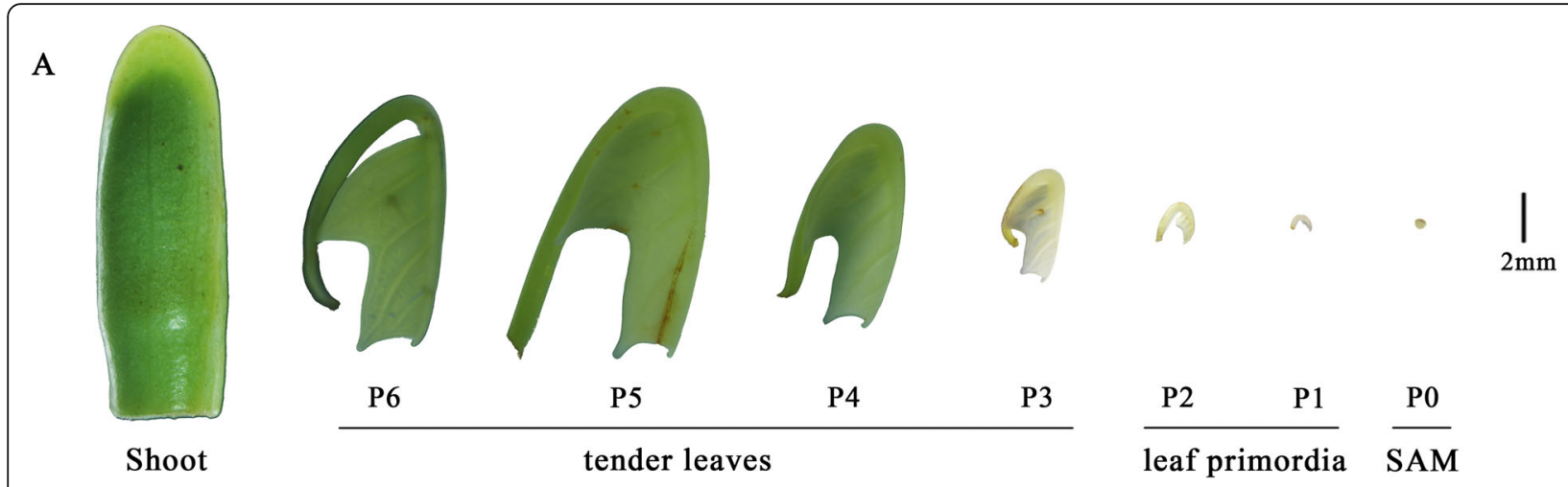

B
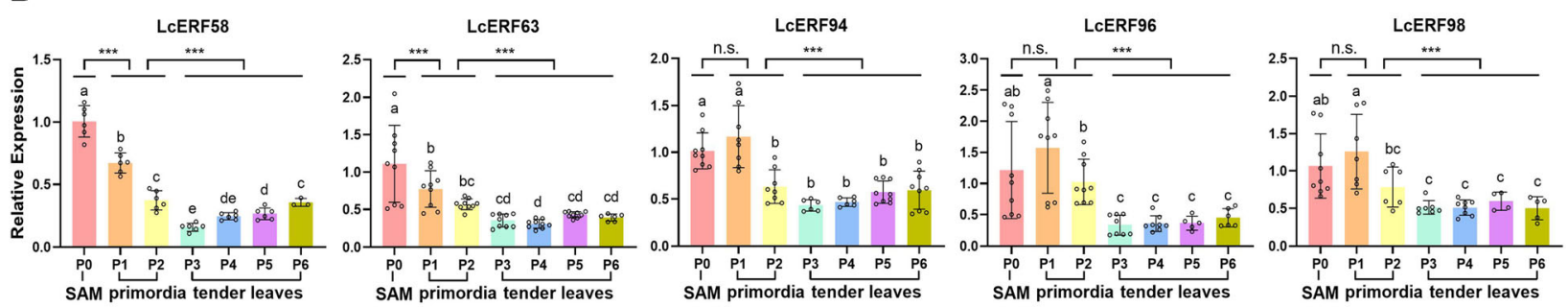

Fig. 6 Dynamic changes in L. chinense leaves at different developmental stages. (A) Morphological anatomy of the shoots under the stereomicroscope. According to the anatomic structure, the developmental stages of leaf were defined as P0 (SAM), P1, P2, P3, P4, P5, and P6 (P1 $\sim$ P2 were the leaf primordia, while P3 P6 were identified as tender leaves). (B) Relative expression of five candidates in different developmental stages. The round dot is the original value, and the error bar shows Std. deviation. For statistical analyses, ANOVA and Duncan tests were used $(p<0.05)$. ${ }^{* * *}$ indicates $P$-value $<0.001$; n.s. indicates no significant)

AP2, RAV and soloist. Global survey of the ERF subfamily was first reported by Sakuma, in which all AtERFs were divided into DREB (group A) and ERF (group B) based on the sequence identities of the DNA-binding domains [18]. Furthermore, Nakano developed Sakuma's classification and subdivided ERF genes into 12 groups according to the genome annotation and phylogenetic analysis of $A$. thaliana and rice [19]. Similarly, in this study, all 104 LcAP2/ERFs were wholly sorted into four subfamilies: ERF, AP2, RAV and soloist, which is consistent with former classifications. Meanwhile, each subfamily of AP2/ERF was provided with an unequal number of proteins in distinct species, while in any case, the ERF subfamily was the largest one, and both the DREB group and the ERF group are of particular interest owing to their involvement in plant responses to stresses [39].

Gene function is mainly dependent on unique features [40]. The "WLG" (Trp-28, Leu-29, and Gly-30) of the AP2/ERF domain is completely conserved in the DREB and ERF subfamilies, and it has also been proven in many other plants, such as Arabidopsis [19], rice [21]' and sorghum [41]. However, the major differentiators of ERF and DREB subfamilies are nonsynonymous substitutions at some specific motifs, i.e., V14 (valine) and E19 (glutamic) are conserved in DREB, while A14 (alanine) and D19 (aspartic) exist in ERF [18]. In many cases, ERF and DREB genes play a role in response to stresses such as drought, salt and low temperature through ABAindependent signaling pathways. DREB proteins, such as CBF1, CBF2, CBF3 [42-44] and DREB1B [45], contain the DNA binding domain that binds to the cis-acting dehydration-responsive element DRE/CRT in the promoters and activates its expression to enhance the abiotic resistance of transgenic plants [46, 47]. While the ERF subfamily genes mainly work to mediate pathogenand disease-related stimuli by integrating multiple signaling pathways, such as the jasmonic acid (JA), ethylene (ET) and salicylic acid (SA) pathways [39]. Evidence shows that ERF proteins bind to the cis-element GCC box (GCCGCC) and directly regulate the expression of pathogenesis-related (PR) genes [48]. However, exceptions also exist that the ERF VII, VIII, and IX groups partially respond to ethylene signal to participate in plant growth and development. For instance, MaERFs are involved in regulating ethylene-mediated fruit ripening of banana [49]. Recent research on pineapple also showed that AcERFs might be positive and key regulators in response to ethylene and induce flowering in pineapple [50], which provided evidence that some ERF genes might be involved in the development of floral organs.

In contrast to ERFs, AP2 subfamily members are considered regulators that help to maintain meristems and regulate organ initiation and growth [51]. Although 

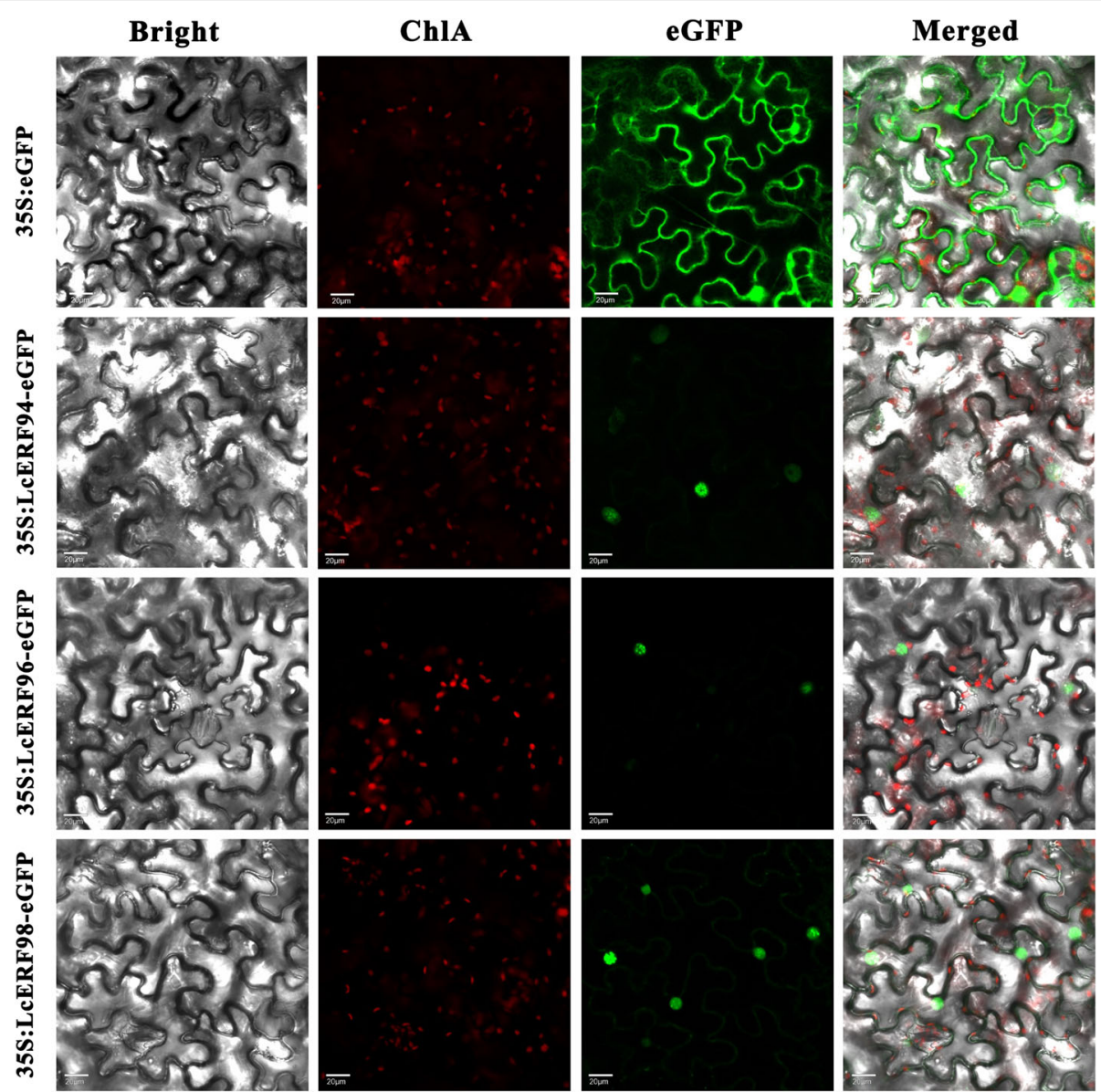

Fig. 7 Subcellular localization of LCERF94/96/98 proteins. The candidates were driven by the 355 promoter. The excitation maximum of GFP was $488 \mathrm{~nm}$, and the excitation maximum of GFP was $509 \mathrm{~nm}$

containing the AP2 domain as well, the AP2 proteins are quite different from the ERFs in terms of conserved domains and gene structure. Conserved motif analysis presented that the amino acid structure of the AP2 domain displayed a low similarity between the AP2 and ERF subfamilies, and "WLG" was converted into "YLG" elements in all AP2 subfamily members [14]. Furthermore, the AP2 genes were interrupted by $6 \sim 11$ introns, which also gives them a variety of functions in the plant development process [16]. In most cases, the AP2 genes respond to plant development and morphogenesis, especially in hormone-mediated morphological development of leaf and flower organs [52-54]. AINTEGUMENTA (ANT) and AINTEGUMENTA-LIKE (AIL) TFs of the AP2 subfamily are expressed in all dividing tissues and have central roles in developmental processes such as embryogenesis [55], root development [56], organ initiation, and growth [57].

Plant leaves are initiated from the shoot apical meristem (SAM), and later grow toward three axes, including the adaxial-abaxial (top-bottom), proximal-distal (base-to-tip) and medio-lateral (central-to-edge) axes [58]. Once the polarity is established, the leaf primordium starts to extend to all sides and finally determines leaf size and shape [59]. In this period, multiple TFs were up-regulated and involved in morphogenesis, such as MYB-domain proteins, KNOTTED1-like homeobox proteins, AUXIN RESPONSE FACTORS, HD-ZIP III genes, basic helix-loop-helix (bHLH) transcription factors and $A P 2 / E R F$, etc. Overexpressed Larix AP2L1 in Arabidopsis leads to enlarged rosette leaves by affecting cell size as well as cell proliferation [60]. The AP2 genes were also involved in the arrangement of phyllotaxy. The PLT3, PLT5 and PLT7 genes acted as key regulators that reshaped the phyllotaxis pattern of $A$. thaliana by regulating AtPIN1 activity [61]. Subsequent research further showed that PLT5 and PLT7 were differentially expressed between $C$. hirsuta and A. thaliana during early leaf development, and expressed ChPLT7 in the simple leaf margin of $A$. thaliana under the $C U C 2$ promoter to cause ectopic leaflet formation [62].

In this study, LcERF94, LcERF96, and LcERF98 showed high similarity with $A N T$ or $A I L$ in their conserved 
domain and gene structure. These three candidates presented high expression levels in early leaf development, and these patterns were consistent with the patterns of ANT and AIL genes from Arabidopsis and Cucurbita moschata [57, 63, 64]. Based on these results, we conjecture that three candidates (LcERF94, LcERF96, and LCERF98) might regulate early leaf development in the same way as the ANT and AIL genes. Among them, $A N T$ genes exhibit the highest expression at the top of shoots, and participate in the initiation, growth and organ size development of higher plant organs by regulating cell proliferation and division [57]. Additionally, AIL genes were detected with high levels in young and actively dividing tissues in Arabidopsis by detecting the relative expression of various tissues [63]. More importantly, after receiving stimulation from growth regulators, the ANTs are regulated positively to maintain cell meristematic competence, thereby modulating the expression of cell growth regulators and promoting organ growth [57].

\section{Conclusions}

In summary, we identified 104 putative AP2/ERF genes in Liriodendron, which were entirely grouped into four major subfamilies. The classification and expression profiling of the AP2/ERF genes provide information for the identification of potential target genes, allowing conjecture regarding the roles of these genes. Furthermore, the STC analysis and dynamic patterns of LcERF94/96/98 in leaf development period indicated that these genes might affect shoot and leaf development. Regardless, this study provides a new perspective for exploring the function of LcERFs in regulating plant growth and development, and three genes identified in this study are good candidates for subsequent functional investigations.

\section{Methods}

\section{Plant materials}

Plant materials were collected from the forest farm attached to Nanjing Forestry University, Jiangsu Province, China $\left(119^{\circ} 13^{\prime} 20^{\prime \prime} E, 32^{\circ} 7^{\prime} 8^{\prime \prime} N\right)$. Sample trees were originally from Lushan, Jiangxi Province $\left(116^{\circ} 0^{\prime} \mathrm{E}, 29^{\circ} 32^{\prime} \mathrm{N}\right)$ (Specimen No. 20010020016, deposited in the specimen room of Nanjing Forestry University) and have been planted in forest farm since 1993. In the middle of spring 2018, various tissues, including shoots, leaf sepal, petal, stamen, pistil, and stem tissues, were removed from mature $L$. chinense trees. In addition, young leaves in distinct developmental periods were also collected from the same trees in the summer of 2019. For each of the samples, no less than three biological replicates were collected, all of which were removed, immediately frozen in liquid nitrogen, and stored at $-80^{\circ} \mathrm{C}$.

\section{Identification and characteristics of the AP2/ERF genes of} L. chinense

The $L$. chinense genome, which was published in 2019, was downloaded from the NCBI genome resource database [29]. Arabidopsis AP2/ERF proteins were obtained from the Plant Transcription Factor database (http://plntfdb.bio.uni-potsdam.de/v3.0/) and utilized for BLASTP searches against genome sequences of $L$. chinense with expected values less than $10^{-3}$. Moreover, the AP2/ERF domain (Pfam accession is PF00847) was downloaded from the Pfam entrance database (http://pfam.xfam.org/) and then used to retrieve AP2/ERF-domain amino acid sequences from all annotated genes of the $L$. chinense genome using the HMMER program (v3.0). In addition to the genome-wide survey, we also used the published transcriptome data (No. SRR8101043, SRR8101042, SRR8101041 and SRR8101040) to determine the integrity and reliability of all AP2/ERF information as much as possible [30].

All candidate AP2/ERF-domain amino acid sequences were assessed based on the presence of the conserved domain using Pfam search (http://pfam.xfam.org/ search\#tabview=tab1) and CDD search (https://www.ncbi. nlm.nih.gov/Structure/bwrpsb/bwrpsb.cgi) procedures. In addition, sequences that incorrectly occupied or even did not carry a complete domain were removed from the list of putative genes. Conserved motifs are essential characteristics of a gene family that perform various functions. Thus, all putative AP2/ERF-domain amino acid sequences were divided into distinct subfamilies according to their motif characteristics.

\section{Phylogenetic analysis}

To determine the evolutionary relationship of all AP2/ ERF sequences, subfamily classification of all AP2/ERF sequences was further confirmed by constructing an unrooted phylogenetic tree with the neighbor-joining (NJ) method. The conserved domain extracted from the whole length of the AP2/ERF sequences was used for multiple sequence alignment using ClustalW with default parameters in the MEGA X software package [65]. The phylogenetic tree was then constructed using the NJ method with 1000 bootstrap replicates in MEGA X. Finally, the network profile of the phylogenetic tree was visualized using Evoview online software (https://www. evolgenius.info/evolview/\#login).

\section{Conserved motif and gene structure analyses}

To investigate the gene structure of the AP2/ERF family, the annotation profile of the $L$. chinense genome was retrieved from the China National GeneBank (CNGB). Information on all exon and intron loci was extracted and later visualized with GSDS (v2.0) (http://gsds.cbi.pku.edu.cn/). In 
parallel with the gene structure surveys, the conserved motifs of the $L$. chinense AP2/ERF family were predicted utilizing MEME (v5.1.1) (http://meme-suite.org/tools/meme) based on the full-length protein sequences with the following parameters: maximum number of motifs of 15; motif sites distributed among sequences with zero or one per sequence model. The results of the motif analysis were then visualized with TBtools software [66].

\section{Series test of cluster (STC) analysis of LCAP2/ERF expression in various tissues}

The expression profiles of various tissues (including bract, sepal, petal, stamen, pistil leaf, shoot, and a mixture of all the floral organs named BSPSP) were obtained from the NCBI database (No. PRJNA559687) and used to demonstrate the expression profiles of all LcAP2/ERF genes [31]. The data preprocessing of sequences was performed mainly in three steps: 3'-trimming, filtering by Phred score and removing low complexity sequences (less than Q20) using the FastQC toolkit. All the filtered clean reads were assigned to the reference genome of Liriodendron chinense using TopHat2 [67]. Programs were run with defaults options except for a maximum size of intron of 5000 base pair. Expression of each gene was further calculated using the fragments per kilobase of exon model per million mapped fragments (FPKM) measurements by Cufflinks [68]. The arithmetic used the default parameters (cufflinks SRR9945430.bam -G genes.gtf -o FPKM.result) [69]. The FPKM values were standardized by Z-score normalization to reflect the expression pattern of all annotated LcAP2/ERF genes. Furthermore, we generated heat maps of all genes using TBtools according to the instructions (parameters: Log Scale, Base 2.0, LogWith 1.0; cluster by row and column) [66].

To discover the genes involved in shoot development, we used STC analysis (STC, series test of cluster) to derive the candidates that were markedly enriched in the shoot-specific cluster. STC analysis was performed using K-means clustering method by STEM program, the number of clusters (K) was 10 and number of random starts was 20. We filter expression values with default parameters, and the correction method was Bonferroni's correction with the significant level was 0.05 [70]. Then cluster analysis and functional annotation of these candidates were performed in GenBank and the TAIR database.

\section{Histological anatomy and morphological observation}

We collected and dissected the shoots in layers to obtain tender leaves at different stages of development and observed them under an OLYMPUS SZX16 stereomicroscope. The developmental stages of leaf were defined as P0 (SAM), P1, P2, P3, P4, P5, and P6 from the innermost to the outermost layer of the buds based on their developmental morphology (P1 P2 were the leaf primordia, while P3 $\sim$ P6 were identified as tender leaves). Moreover, materials from different stages were collected and immediately frozen in liquid nitrogen and stored at $-80^{\circ} \mathrm{C}$ for RNA extraction.

\section{RNA extraction and RT-qPCR analysis of AP2/ERF genes} Total RNA was extracted from samples with the RNAprep pure kit (Tiangen, Beijing, China) according to the manufacturer's instructions. Then, cDNA was synthesized from $500 \mathrm{ng}$ of total RNA using PrimeScirptTM RT Master Mix (TaKaRa, Dalian, China) in a $10-\mu \mathrm{L}$ reaction volume according to the instructions. Before polymerase chain reaction, the cDNA was diluted $1 / 10$ with mother liquor made with deionized water to reduce systematic error. Then, specific primers for the LcERF genes were designed with Oligo 7.0 software adhering to the instructions (see Additional file 2: Table S2). RTqPCR was performed on a StepOnePlus ${ }^{\text {Tw }}$ System (Applied Biosystems) with $10-\mu \mathrm{L}$ reaction mixtures containing $5 \mu \mathrm{L}$ of $2 \times$ SYBR Premix Ex Taq, $0.2 \mu \mathrm{L}$ of $50 \times$ ROX Reference Dye (TaKaRa, Dalian, China), primers, and cDNA. The relative expression of the $L c E R F$ genes was calculated using the $\Delta \Delta C_{T}$ method. Three biological replicates were set per sample, and each sample had three technical replicates. In addition, the LcActin97 gene was used as a reference gene in this process, which has been shown to be reliable in $L$. chinense [71]. In addition, all the RT-qPCR relative expression data were further analyzed using SPSS 11.5, and ANOVA and Duncan's test were also performed to test the significant differences among various samples.

\section{Subcellular localization assay}

We further constructed recombinant proteins that fused the eGFP marker with the LCERF proteins in the Cterminus. The pBI121-eGFP vector (GUS was replaced with GFP in the original PBI121 vector) was restricted by the XbarI and BamHI enzymes along with insertion of the recombinant proteins into the digested vector. Subsequently, the recombinant plasmid sequences were verified by the Sanger sequencing platform and then transferred into Agrobacterium tumefaciens (GV3101). After overnight incubation at $28^{\circ} \mathrm{C}$, the OD600 value of the bacterial solution reached $0.6 \sim 0.8$. We collected recombinant bacteria by centrifugation at $4000 \mathrm{rpm}$ and resuspended the bacteria in infection buffer $(10 \mathrm{mM}$ $\mathrm{MgCl}_{2}, 10 \mathrm{mM}$ MES, $150 \mu \mathrm{M}$ HO-AS, with a final $\mathrm{pH}=$ 5.6). Moreover, the helper vector $\mathrm{P} 19$ was subjected to the same treatment and mixed equally with ERF. The resuspended mixtures were injected into tender tobacco leaves. After $1 \sim 2$ days of coculture, we observed and recorded the GFP fluorescence signal under a laser confocal microscope. 


\section{Abbreviations}

TFs: Transcription factors; AP2/EREBPs: APETALA2/ethylene responsive element-binding proteins; ERF: Ethylene responsive element binding factor; DREB: Dehydration responsive element binding factor; RAV: Related to ABI3/ VP; ANT: AINTEGUMENTA; AIL: AINTEGUMENTA-Like; SAM: Shoot Apical Meristem; DRN: DORNROESCHEN; DRNL: DRN-LIKE; FPKM: Fragments Per Kilobase Million; GFP: Green fluorescent protein

\section{Supplementary Information}

The online version contains supplementary material available at https://doi. org/10.1186/s12864-021-08119-7.

Additional file 1: Table S1. List of the 104 AP2/ERF genes identified in L. chinense.

Additional file 2: Table S2. List of primer sequences in this study.

\section{Acknowledgments}

The authors would like to thank Dr. Wenjie Ding for advice on data analysis; Dr. Huanhuan Liu for assistance in experiments. In addition, the authors also like to thank other colleagues in the laboratory for their help.

\section{Authors' contributions}

H. L. designed the research. Y. Z. and Z. H. analyzed the data and prepared Figs. Y. Z., Z. H., Z. T., Y. S., C. Z., S. W., and L. Y. performed the experiments. Y. Z. and Z. H. did final editing of the manuscript. Y. Z. and Z. H. have contributed equally to this work. All authors contributed to the article and approved the submitted version.

\section{Funding}

The design and experiments of this study were supported by the National Natural Science Foundation of China (31770718 and 31470660) and the Priority Academic Program Development of Jiangsu Higher Education Institutions (PAPD). The funding bodies played no role in the design of the study and collection, analysis, and interpretation of data and in writing the manuscript.

\section{Availability of data and materials}

All data presented in this study are provided either in the manuscript or additional files. The datasets generated and analysed during the current study are available in the NCBI with the accession numbers SRR8101043, SRR8101042, SRR8101041, SRR8101040 and PRJNA559687.

\section{Declarations}

Ethics approval and consent to participate

The authors confirm that the material collection presented here were conducted in accordance with the wild plant care regulations and natural reserves regulations set forth by the Decree of the state council of the People's Republic of China.

\section{Consent for publication}

Not applicable.

\section{Competing interests}

The authors declare that they have no competing interests

Received: 28 July 2020 Accepted: 23 October 2021

Published online: 08 November 2021

\section{References}

1. Ma Y, Wang F, Guo J, Zhang XS. Rice OsAS2 gene, a member of LOB domain family, functions in the regulation of shoot differentiation and leaf development. J Plant Biol. 2009:52(5):374-81. https://doi.org/10.1007/s123 74-009-9048-4.

2. Barkoulas M, Hay A, Kougioumoutzi E, Tsiantis M. A developmental framework for dissected leaf formation in the Arabidopsis relative Cardamine hirsuta. Nat Genet. 2008:40(9):1136-41. https://doi.org/10.1038/ ng.189.
3. Schruff MC, Spielman M, Tiwari S, Adams S, Fenby N, Scott RJ. The AUXIN RESPONSE FACTOR 2 gene of Arabidopsis links auxin signalling, cell division, and the size of seeds and other organs. Development. 2005;133(2):251-61. https://doi.org/10.1242/dev.02194.

4. Gutierrez C. The Arabidopsis cell division cycle. The Arabidopsis Book, The Am Soc Plant Biologists. 2009:7:1-19. https://doi.org/10.1199/tab.0120.

5. Takada S, Hibara K, Ishida T, Tasaka M. The CUP-SHAPED COTYLEDON1 gene of Arabidopsis regulates shoot apical meristem formation. Development. 2001;128(7):1127-35. https://doi.org/10.1242/dev.128.7.1127.

6. Barton MK, Poethig RS. Formation of the shoot apical meristem in Arabidopsis thaliana: an analysis of development in the wild-type and in the SHOOT MERISTEMLESS mutant. Development. 1993;119(3):823-31. https:// doi.org/10.1242/dev.119.3.823.

7. Wurschum T, Groshardt R, Laux T. APETALA2 regulates the stem cell niche in the Arabidopsis shoot meristem. Plant Cell. 2006;18(2):295-307. https:// doi.org/10.1105/tpc.105.038398

8. Ohto M, Floyd SK, Fischer RL, Goldberg RB, Harada JJ. Effects of APETALA2 on embryo, endosperm, and seed coat development determine seed size in Arabidopsis. Sex Plant Reprod. 2009;22(4):277-89. https://doi.org/10.1007/ s00497-009-0116-1.

9. McGrath KC, Dombrecht B, Manners JM, Schenk PM, Edgar Cl, Maclean DJ, et al. Repressor- and activator-type ethylene response factors functioning in jasmonate signaling and disease resistance identified via a genome-wide screen of Arabidopsis transcription factor gene expression. Plant Physiol. 2005;139(2):949-59. https://doi.org/10.1104/pp.105.068544.

10. Yamaguchishinozaki K, Shinozaki K. Transcriptional regulatory networks in cellular responses and tolerance to dehydration and cold stresses. Plant Biol. 2006;57(1):781-803. https://doi.org/10.1146/annurev.arplant.57.032905.1 05444.

11. Marschmartinez N, Greco R, Becker JD, Dixit S, Bergervoet JHW, Karaba A, et al. BOLITA, an Arabidopsis AP2/ERF-like transcription factor that affects cell expansion and proliferation/differentiation pathways. Plant Mol Biol. 2006;62(6):825-43. https://doi.org/10.1007/s11103-006-9059-1.

12. Du D, Hao R, Cheng T, Pan H, Yang W, Wang J, et al. Genome-wide analysis of the AP2/ERF gene family in Prunus mume. Plant Mol Biol Report. 2013; 31(3):741-50. https://doi.org/10.1007/s11105-012-0531-6.

13. Agarwal G, Garg V, Kudapa H, Doddamani D, Pazhamala LT, Khan AW, et al. Genome-wide dissection of AP2/ERF and HSP90 gene families in five legumes and expression profiles in chickpea and pigeonpea. Plant Biotechnol J. 2016;14(7):1563-77. https://doi.org/10.1111/pbi.12520.

14. Liu M, Sun W, Ma Z, Zheng T, Huang L, Wu Q, et al. Genome-wide investigation of the AP2/ERF gene family in tartary buckwheat (Fagopyum Tataricum). BMC Plant Biol. 2019;19(1):342. https://doi.org/10.1186/s12870-01 9-1951-3

15. Jofuku KD, Bg DB, Van Montagu M, Okamuro JK. Control of Arabidopsis flower and seed development by the homeotic gene APETALA2. Plant Cell. 1994:6(9):1211-25. https://doi.org/10.1105/tpc.6.9.1211.

16. Licausi F, Ohmetakagi M, Perata P. APETALA2/ethylene responsive factor (AP2/ERF) transcription factors: mediators of stress responses and developmental programs. New Phytol. 2013;199(3):639-49. https://doi.org/1 $0.1111 /$ nph.12291.

17. Riechmann JLME. The AP2/EREBP family of plant transcription factors. Biol Chem. 1998:379(6):633-46. https://doi.org/10.1515/bchm.1998.379.6.633.

18. Sakuma Y, Liu Q, Dubouzet JG, Abe H, Shinozaki K, Yamaguchishinozaki K. DNA-binding specificity of the ERF/AP2 domain of Arabidopsis DREBs, transcription factors involved in dehydration- and cold-inducible gene expression. Biochem Biophys Res Commun. 2002;290(3):998-1009. https:// doi.org/10.1006/bbrc.2001.6299.

19. Nakano T, Suzuki K, Fujimura T, Shinshi H. Genome-wide analysis of the ERF gene family in Arabidopsis and Rice. Plant Physiol. 2006;140(2):411-32. https://doi.org/10.1104/pp.105.073783.

20. Sakuma Y, Maruyama K, Osakabe Y, Qin F, Seki M, Shinozaki K, et al. Functional analysis of an Arabidopsis transcription factor, DREB2A, involved in drought-responsive gene expression. Plant Cell. 2006;18(5):1292-309. https://doi.org/10.1105/tpc.105.035881.

21. Sharoni AM, Nuruzzaman M, Satoh K, Shimizu T, Kondoh H, Sasaya T, et al. Gene structures, classification, and expression models of the AP2/EREBP transcription factor family in Rice. Plant Cell Physiol. 2011:52(2):344-60. https://doi.org/10.1093/pcp/pcq196.

22. Liu Q. Two transcription factors, DREB1 and DREB2, with an EREBP/AP2 DNA binding domain separate two cellular signal transduction pathways in 
drought- and low-temperature-responsive gene expression, respectively, in Arabidopsis. Plant Cell. 1998;10(8):1391-406. https://doi.org/10.1105/tpc.10. 8.1391.

23. Hao D, Ohmetakagi M, Sarai A. Unique mode of GCC box recognition by the DNA-binding domain of ethylene-responsive element-binding factor (ERF domain) in plant. J Biol Chem. 1998;273(41):26857-61. https://doi.org/1 0.1074/jbc.273.41.26857.

24. Welsch R, Maass D, Voegel T, Dellapenna D, Beyer P. Transcription factor RAP2.2 and its interacting partner SINAT2: stable elements in the Carotenogenesis of Arabidopsis leaves. Plant Physiol. 2007;145(3):1073-85. https://doi.org/10.1104/pp.107.104828.

25. Chandler JW. Class VIIIb APETALA2 ethylene response factors in plant development. Trends Plant Sci. 2017;23(2):151-62. https://doi.org/10.1016/j. tplants.2017.09.016.

26. Der Graaff EV, Den Dulkras A, Hooykaas PJJ, Keller B. Activation tagging of the LEAFY PETIOLE gene affects leaf petiole development in Arabidopsis thaliana. Development. 2000;127(22):4971-80. https://doi.org/10.1242/dev.12 7.22.4971.

27. Banno H, Ikeda Y, Niu Q, Chua N. Overexpression of Arabidopsis ESR1 induces initiation of shoot regeneration. Plant Cell. 2001;13(12):2609-18. https://doi.org/10.1105/tpc.010234.

28. Aida M, Beis D, Heidstra R, Willemsen V, Blilou I, Galinha C, et al. The PLETHORA genes mediate patterning of the Arabidopsis root stem cell niche. Cell. 2004;119(1):109-20. https://doi.org/10.1016/j.cell.2004.09.018.

29. Chen J, Hao Z, Guang X, Zhao C, Wang P, Xue L, et al. Liriodendron genome sheds light on angiosperm phylogeny and species-pair differentiation. Nat Plants. 2019:5(1):18-25. https://doi.org/10.1038/s41477-01 8-0323-6.

30. Ma J, Wei L, Li J, Li H: The Analysis of Genes and Phytohormone Metabolic Pathways Associated with Leaf Shape Development in Liriodendron chinense via De Novo Transcriptome Sequencing. Genes 2018, 9(12), 9, 12, https:// doi.org/10.3390/genes9120577.

31. Tu Z, Shen Y, Wen S, Zong Y, Li H. Alternative splicing enhances the transcriptome complexity of Liriodendron chinense. Front Plant Sci. 2020;11: 1460. https://doi.org/10.3389/fpls.2020.578100.

32. Matsuo N, Makino M, Banno H. Arabidopsis ENHANCER OF SHOOT REGENERATION (ESR) 1 and ESR2 regulate in vitro shoot regeneration and their expressions are differentially regulated. Plant Sci. 2011;181(1):39-46. https://doi.org/10.1016/j.plantsci.2011.03.007.

33. Zhu Y, Li Y, Zhang S, Zhang X, Yao J, Luo Q, et al. Genome-wide identification and expression analysis reveal the potential function of ethylene responsive factor gene family in response to Botrytis cinerea infection and ovule development in grapes (Vitis vinifera L.). Plant Biol. 2019; 21(4):571-84. https://doi.org/10.1111/plb.12943.

34. Zhuang J, Cai B, Peng R, Zhu B, Jin X, Xue Y, et al. Genome-wide analysis of the AP2/ERF gene family in Populus trichocarpa. Biochem Biophys Res Commun. 2008;371(3):468-74. https://doi.org/10.1016/j.bbrc.2008.04.087.

35. Shu Y, Liu Y, Zhang J, Song L, Guo C. Genome-wide analysis of the AP2/ERF superfamily genes and their responses to abiotic stress in Medicago truncatula. Front Plant Sci. 2016;6:1247. https://doi.org/10.3389/fpls.2015. 01247.

36. Zhang Z, Li X. Genome-wide identification of AP2/ERF superfamily genes and their expression during fruit ripening of Chinese jujube. Sci Rep. 2018; 8(1). https://doi.org/10.1038/s41598-018-33744-w.

37. Li $X$, Tao $S$, Wei $S$, Ming M, Huang $X$, Zhang $S$, et al. The mining and evolutionary investigation of AP2/ERF genes in pear (Pyrus). BMC Plant Biol. 2018;18(1):46. https://doi.org/10.1186/s12870-018-1265-X.

38. Zhang S, Zhu C, Lyu Y, Chen Y, Zhang Z, Lai Z, et al. Genome-wide identification, molecular evolution, and expression analysis provide new insights into the APETALA2/ethylene responsive factor (AP2/ERF) superfamily in Dimocarpus longan Lour. BMC Genomics. 2020;21(1):62. https://doi.org/10.1186/s12864-020-6469-4

39. Rehman S, Mahmood T. Functional role of DREB and ERF transcription factors: regulating stress-responsive network in plants. Acta Physiol Plant. 2015;37(9):37-9. https://doi.org/10.1007/s11738-015-1929-1.

40. Liu L, White MJ, MacRae TH. Transcription factors and their genes in higher plants: functional domains, evolution and regulation. Eur J Biochem. 1999; 262(2):247-57. https://doi.org/10.1046/j.1432-1327.1999.00349.x.

41. Mathur S, Priyadarshini SS, Singh V, Vashisht I, Jung K-H, Sharma R, et al. Comprehensive phylogenomic analysis of ERF genes in sorghum provides clues to the evolution of gene functions and redundancy among gene family members. 3. Biotech. 2020;10(3):1-16. https://doi.org/10.1007/s13205020-2120-y.

42. Zhou L, Li J, He Y, Liu Y, Chen H. Functional characterization of SmCBF genes involved in abiotic stress response in eggplant (Solanum melongena). Sci Hortic. 2018;233:14-21. https://doi.org/10.1016/j.scienta.201 8.01.043.

43. G-q S, Gao X. Transcriptomic changes reveal gene networks responding to the overexpression of a blueberry DWARF AND DELAYED FLOWERING 1 gene in transgenic blueberry plants. BMC Plant Biol. 2017;17(1). https://doi. org/10.1186/s12870-017-1053-Z

44. Siddiqua M, Nassuth A. Vitis CBF1 and Vitis CBF4 differ in their effect on Arabidopsis abiotic stress tolerance, development and gene expression. Plant Cell Environ. 2011;34(8):1345-59. https://doi.org/10.1111/j.1365-3040.2 011.02334.x

45. Movahedi S, Sayed Tabatabaei, B.E., Alizade, H. et al. : Constitutive expression of Arabidopsis DREB1B in transgenic potato enhances drought and freezing tolerance. Biol Plant 2012, 56:37-42, 1, https://doi.org/10.1007/ s10535-012-0013-6.

46. Lucas S, Durmaz E, Akpinar BA, Budak H. The drought response displayed by a DRE-binding protein from Triticum dicoccoides. Plant Physiol Biochem. 2011:49(3):346-51. https://doi.org/10.1016/j.plaphy.2011.01.016.

47. Liu Y, Zhao T, Liu J, Liu W, Liu Q, Yan Y, et al. The conserved Ala37 in the ERF/AP2 domain is essential for binding with the DRE element and the GCC box. FEBS Lett. 2006;580(5):1303-8. https://doi.org/10.1016/j.febslet.2006.01. 048.

48. Zarei AKA, Younessi P, Montiel G, Champion A, Memelink J. Two GCC boxes and AP2/ERF-domain transcription factor ORA59 in jasmonate/ethylenemediated activation of the PDF1.2 promoter in Arabidopsis. Plant Mol Biol. 2011;75(4-5):321-31. https://doi.org/10.1007/s11103-010-9728-y.

49. Xiao YY, Chen JY, Kuang JF, Shan W, Xie H, Jiang YM, et al. Banana ethylene response factors are involved in fruit ripening through their interactions with ethylene biosynthesis genes. J Exp Bot. 2013;64(8):2499-510. https:// doi.org/10.1093/jxb/ert108.

50. Zhang H, Pan X, Liu S, Lin W, Li Y, Zhang X. Genome-wide analysis of AP2/ ERF transcription factors in pineapple reveals functional divergence during flowering induction mediated by ethylene and floral organ development. Genomics. 2021;113(2):474-89. https://doi.org/10.1016/j.ygeno.2020.10.040,

51. Mudunkothge JS, Krizek BA. Three Arabidopsis AIL/PLT genes act in combination to regulate shoot apical meristem function. Plant J. 2012;71(1): 108-21. https://doi.org/10.1111/j.1365-313X.2012.04975.x.

52. Rashotte AM, Mason MG, Hutchison CE, Ferreira F, Schaller GE, Kieber JJ. A subset of Arabidopsis AP2 transcription factors mediates cytokinin responses in concert with a two-component pathway. Proc Natl Acad Sci U S A. 2006;103(29):11081-5. https://doi.org/10.1073/pnas.0602038103.

53. Horstman A, Willemsen V, Boutilier K, Heidstra R. AINTEGUMENTA-LIKE proteins: hubs in a plethora of networks. Trends Plant Sci. 2014;19(3):146-57. https://doi.org/10.1016/j.tplants.2013.10.010.

54. Elliott RC, Betzner AS, Huttner E, Oakes MP, Tucker W, Gerentes D, et al. AINTEGUMENTA, an APETALA2-like gene of Arabidopsis with pleiotropic roles in ovule development and floral organ growth. Plant Cell. 1996;8(2): 155-68. https://doi.org/10.1105/tpc.8.2.155.

55. Boutilier K, Offringa R, Sharma VK, Kieft H, Ouellet T, Zhang L, et al. Ectopic expression of BABY BOOM triggers a conversion from vegetative to embryonic growth. Plant Cell. 2002;14(8):1737-49. https://doi.org/10.1105/ tpc.001941.

56. Galinha C, Hofhuis H, Luijten M, Willemsen V, Blilou I, Heidstra R, et al. PLETHORA proteins as dose-dependent master regulators of Arabidopsis root development. Nature. 2007;449(7165):1053-7. https://doi.org/10.1038/ nature06206.

57. Mizukami Y, Fischer RL. Plant organ size control: AINTEGUMENTA regulates growth and cell numbers during organogenesis. Proc Natl Acad Sci U S A. 2000;97(2):942-7. https://doi.org/10.1073/pnas.97.2.942.

58. Satterlee JW, Scanlon MJ. Coordination of leaf development across developmental axes. Plants. 2019;8(10):433. https://doi.org/10.3390/plants81 00433.

59. Wang $\mathrm{H}$, Kong F, Zhou C. From genes to networks: the genetic control of leaf development. J Integr Plant Biol. 2021;63(7):1181-96. https://doi.org/1 0.1111/jipb.13084.

60. Li A, Zhou Y, Jin C, Song W, Chen C, Wang C. LaAP2L1, a heterosisassociated AP2/EREBP transcription factor of Larix, increases organ size and 
final biomass by affecting cell proliferation in Arabidopsis. Plant Cell Physiol. 2013;54(11):1822-36. https://doi.org/10.1093/pcp/pct124.

61. Prasad K, Grigg SP, Barkoulas M, Yadav RK, Sanchez-Perez GF, Pinon V, et al. Arabidopsis PLETHORA transcription factors control phyllotaxis. Curr Biol. 2011;21(13):1123-8. https://doi.org/10.1016/j.cub.2011.05.009.

62. Gan X, Hay A, Kwantes M, Haberer G, Hallab A, loio RD, et al. The Cardamine hirsuta genome offers insight into the evolution of morphological diversity. Nat Plants. 2016;2(11):16167. https://doi.org/10.1038/nplants.2016.167.

63. Nolewilson S, Tranby TL, Krizek BA. AINTEGUMENTA-like (AIL) genes are expressed in young tissues and may specify meristematic or divisioncompetent states. Plant Mol Biol. 2005;57(5):613-28. https://doi.org/10.1007/ s11103-005-0955-6.

64. Miao L, Li S-Z, Shi A-K, Li Y-S, He C-X, Yan Y, et al. Genome-wide analysis of the AINTEGUMENTA-like (AIL) transcription factor gene family in pumpkin (Cucurbita moschata Duch.) and CmoANT1.2 response in graft union healing. Plant Physiol Biochem. 2021;162:706-15. https://doi.org/10.1016/j. plaphy.2021.03.036.

65. Kumar S, Stecher G, Li M, Knyaz C, Tamura K. MEGA X: molecular evolutionary genetics analysis across computing platforms. Mol Biol Evol. 2018;35(6):1547-9. https://doi.org/10.1093/molbev/msy096.

66. Chengjie Chen HC, Zhang Y, Thomas HR, Frank MH, He Y, Xia R. TBtools: an integrative toolkit developed for interactive analyses of big biological data. Mol Plant. 2020;13(8):1194-202. https://doi.org/10.1016/j.molp.2020.06.009.

67. Kim D, Pertea G, Trapnell C, Pimentel H, Kelley R, SL S. TopHat2: accurate alignment of transcriptomes in the presence of insertions, deletions and gene fusions. Genome Biol. 2013;14(4):R36.

68. Trapnell C, Roberts A, Goff L, Pertea G, Kim D, Kelley DR, et al. Differential gene and transcript expression analysis of RNA-seq experiments with TopHat and cufflinks. Nat Protoc. 2012;7(3):562-78. https://doi.org/10.1038/ nprot.2012.016.

69. Brinkman DL, Jia X, Potriquet J, Kumar D, Dash D, Kvaskoff D, et al, Transcriptome and venom proteome of the box jellyfish Chironex fleckeri. BMC Genomics. 2015:16(1):407. https://doi.org/10.1186/s12864-015-1568-3.

70. Ernst J, Nau GJ, Barjoseph Z. Clustering short time series gene expression data. Bioinformatics. 2005;21(1):159-68. https://doi.org/10.1093/bioinforma tics/bti1022.

71. Tu Z, Hao Z, Zhong W, Li H. Identification of Suitable Reference Genes for RT-qPCR Assays in Liriodendron chinense (Hemsl.) Sarg. Forests. 2019;10(5): 441

\section{Publisher's Note}

Springer Nature remains neutral with regard to jurisdictional claims in published maps and institutional affiliations.

Ready to submit your research? Choose BMC and benefit from:

- fast, convenient online submission

- thorough peer review by experienced researchers in your field

- rapid publication on acceptance

- support for research data, including large and complex data types

- gold Open Access which fosters wider collaboration and increased citations

- maximum visibility for your research: over $100 \mathrm{M}$ website views per year

At BMC, research is always in progress.

Learn more biomedcentral.com/submissions 